\title{
Dermatologie 2006 - Ein Querschnittsfach im Wandel
}

\author{
Wissenschaftliches Symposium anlässlich des $\mathbf{8 0}$. Geburtstages von Herrn \\ Prof. Dr. med. O. P. Hornstein, durchgeführt am 28. 1. 2006 in Erlangen \\ P. von den Driesch
}

Dermatology 2006 - A Broad Changing Topic

Anlässlich des 80. Geburtstages des früheren Direktors der Universitäts-Hautklinik des Universitätsklinikums Erlangen, Herrn Prof. Dr. med. O. P. Hornstein, versammelten sich unter der Leitung von Priv.-Doz. Dr. Vera Mahler, Prof. Dr. G. Schuler und Prof. Dr. P. von den Driesch seine Schüler am 28.1.2006 in Erlangen, um den Jubilar zu ehren und die Breite des dermatologischen Fachbereichs mit einer Reihe von Vorträgen darzustellen.

Herr Prof. Dr. med. W. Meinhof, Dormitz, zeigte in einem historisch geprägten Referat, wie sich aus biochemischer Forschung heraus das Wissen um die Porphyrine und die von ihnen ausgelösten Erkrankungen der Porphyrien im Laufe der Jahrzehnte weiterentwickelt haben.

Herr Prof. Dr. med. D. Djawari, Heilbronn, wies in seinem Beitrag auf die Notwendigkeit der Früh-Intervention bei kapillären Hämangiomen des Säuglingsalters hin. Hier werde manchmal durch das in den Lehrbüchern immer noch zu findende zu lange Zuwarten bei diesen Läsionen häufig lediglich Zeit verloren.

Herr Prof. Dr. med. E. Haneke, Freiburg, zeigte in seinem Vortrag die vielfältigen Möglichkeiten, die die Dermatologie als operatives Fach in der heutigen Zeit bietet.

Herr Prof. Dr. med. P. von den Driesch, Stuttgart, wies in seinem Vortrag über Neutrophile Dermatosen darauf hin, dass beispielsweise bei der Psoriasis die Besonderheiten einer Neutrophilenaktivierenden Entzündung zu wenig beachtet werden. Er vertrat die Überzeugung, dass bei der Psoriasis erfolgreich eingesetzte Medikamente häufig beides, T-Lymphozyten und neutrophile Granulozyten, in ihren Funktionen behindern.
Herr Prof. Dr. med. M. Simon, Erlangen, beschäftigte sich in einem Übersichtsreferat mit der Bedeutung von zytotoxischen T-ZellLymphomen und Natural-Killerzell-Lymphomen. Diese häufig als aggressiv wachsende isolierte Tumoren beginnenden Entitäten weisen im Vergleich zu anderen kutanen Lymphomen oft ein aggressives Verhalten auf.

Herr Dr. med. B. Salzer, Heilbronn, zeigte anhand neuester Studienergebnisse, dass professionell durchgeführte NeurodermitisSchulungen, die auch in Praxen durchgeführt werden können, einen erheblichen positiven Einfluss auf den Verlauf des atopischen Ekzems, insbesondere bei Kindern, haben können.

Herr Priv.-Doz. Dr. med. E. Jecht, Nürnberg, zeigte am Beispiel der Psoriasis, dass auch heute noch psychosomatische Aspekte in der Therapie vernachlässigt werden.

Herr Prof. Dr. med. M. Gruschwitz, Kümmersbruck, zeigt in seinem Vortrag zur Pathogenese der progressiven systemischen Sklerodermie, dass der entscheidende pathogenetische Prozess, die zunehmende Sklerose des Gewebes, von einer komplexen Interaktion der ortsständigen Zellen im Sinne einer immunologischen Reaktion begleitet ist.

Herr Priv.-Doz. Dr. med. G. Reimer, Augsburg, gab eine Übersicht über den aktuellen Stand der Diagnostik mittels des Nachweises antinukleärer Antikörper in der dermatologischen Diagnostik. Diese Laboruntersuchungen haben sich zu einem unverzichtbaren Test bezogen auf die Diagnose und Prognose-Bestimmung bei einer Reihe von autoimmunbedingten Systemerkrankungen, wie den Kollagenosen, erwiesen.

Institutsangaben

Klinik für Dermatologie und Allergologie

Korrespondenzadresse

Prof. Dr. med. Peter von den Driesch · Direktor der Klinik für Dermatologie und Allergologie .

Krankenhaus Bad Cannstatt · Prießnitzweg 24 · 70374 Stuttgart · E-mail: PDriesch@kbc-intern.de

Bibliografie

Akt Dermatol 2006; 32: 270-271 @ Georg Thieme Verlag KG Stuttgart · New York

DOI 10.1055/s-2006-925365 · ISSN 0340-2541 


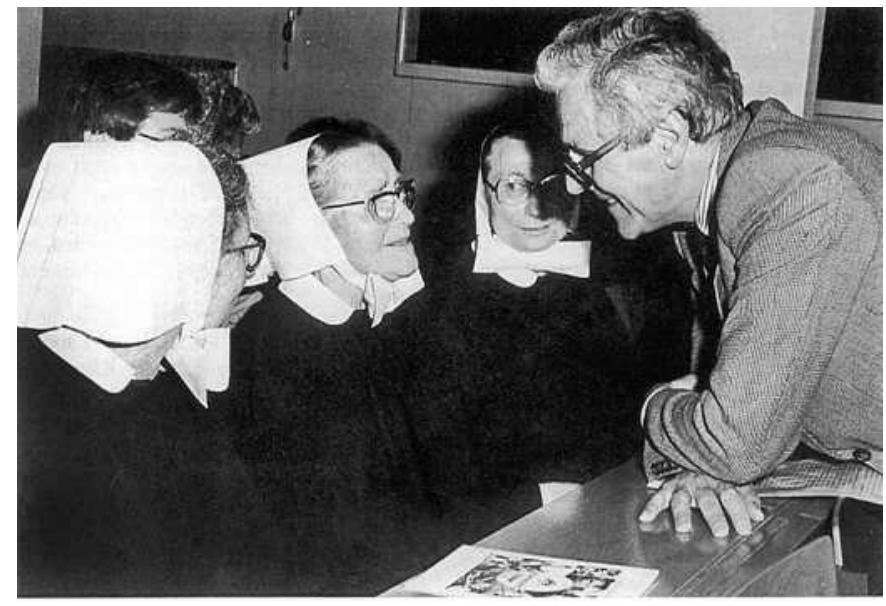

Abb. 1 Professor Hornstein im Gespräch mit früheren Diakonissenschwestern (1976 anläßlich seines 50. Geburtstages).

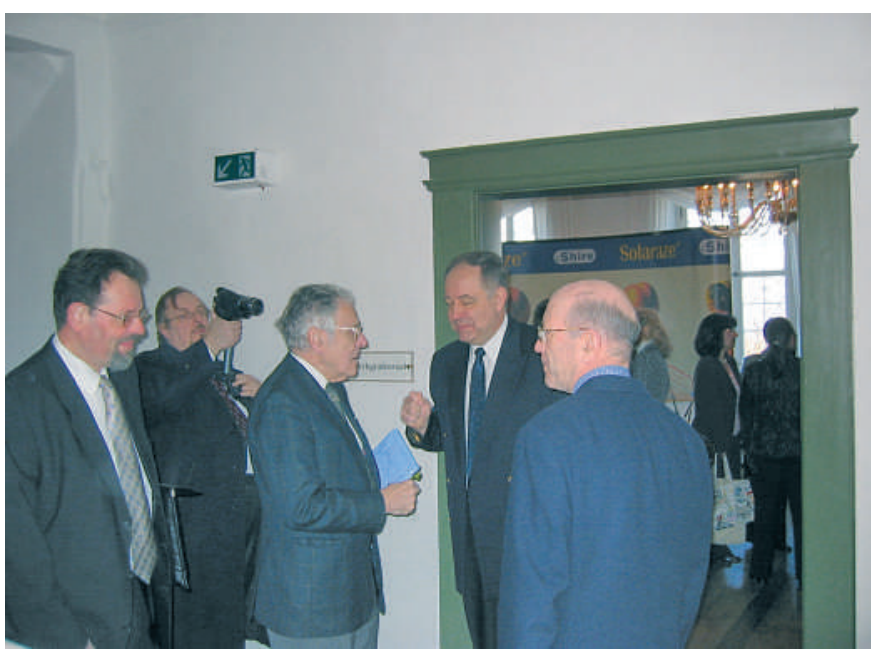

Abb. 2 Professor Hornstein im Kreise seiner Kollegen am Geburtstagssymposium vom 28. Januar 2006.

Herr Prof. Dr. med. F. Kiesewetter, Erlangen, wies in seinem Vortrag auf das oft unterschätzte Problem eines Klimakterium virile und die Problematik seiner Diagnostik und Therapie hin.

Herr Priv.-Doz. Dr. med. J. Keller, Stuttgart, zeigte in einem Übersichtsreferat die Möglichkeiten der ästhetischen dermatologischen Therapie, die unter Einsatz konservativer und operativer Möglichkeiten heutzutage in vielen Fällen für die Patienten befriedigende Ergebnisse erzielen kann.

Herr Prof. Dr. med. G. Schuler, Erlangen, gab einen Überblick über die aktuelle Forschung an der dermatologischen Universitätsklinik in Erlangen. Im Focus stehen die Möglichkeiten einer gezielten Immunintervention mittels dendritischer Zellen bei onkologischen und autoimmunbedingten Erkrankungen.
Als Ausklang der gelungenen Veranstaltung gab Herr Prof. Dr. med. F. Weidner, Stuttgart, im Zusammenspiel mit Herrn Dr. med. H. Heyd, Weißenburg, dem Jubilar ein Geburtstagskonzert.

Alle Schüler von Prof. Dr. med. O. P. Hornstein bedankten sich für die enorme Breite der Forschung, die unter der Führung des Jubilars an der Dermatologischen Universitätsklinik Erlangen ermöglicht und gefördert wurde, sowie für die Breite und Tiefe der durch Prof. Dr. med. O. P. Hornstein gelehrten klinischen Dermatologie.

\section{Lebenslauf Otto P. Hornstein}

Geboren am 22.1.1926 in München

1944:

Reifeprüfung Melanchthon-Gymnasium Nürnberg

bis 1945:

1946 - 1950:

Kriegsdienst mit Gefangenschaft

Studium der Medizin an den Universitäten Erlangen, Würzburg und München; Staatsexamen Dezember 1950 LMU München

1951: Promotion zum Dr. med. (magna cum laude) Universität Würzburg

1951-1953: $\quad$ Pathologisch-anatomische Weiterbildung in Regensburg, Würzburg und Zürich

Ende 1953-1958: Wissenschaftlicher Assistent Univ.-Hautklinik Würzburg

1958: Facharzt für Dermatologie, Habilitation (Februar 1958), Erteilung der Lehrbefugnis für Dermatologie und Venerologie, Ernennung zum Priv.-Doz. (Universität Würzburg)

$1958-1962$

Wiss. Assistent an der Univ.-Hautklinik Bonn, anschließend Europarat-Stipendium für experimetelle Biologie (Universität Uppsala, Inst. f. Zoophysiologie)

1963 - 1967: $\quad$ Oberarzt und apl. Prof. (1964) an der Universitäts-Hautklinik Düsseldorf

seit 1.5.1967: o. Prof. für Dermatologie und Venerologie Direktor der Dermatologischen Klinik der FAU Erlangen-Nürnberg

1977:

Ablehnung eines Rufes auf den Lehrstuhl für Dermatologie an der Universität Bonn

1.4.1995: $\quad$ Emeritierung 